\title{
Review
}

Elisa De Paolis, Giovanni Luca Scaglione, Maria De Bonis, Angelo Minucci and Ettore Capoluongo*

\section{CYP24A1 and SLC34A1 genetic defects associated with idiopathic infantile hypercalcemia: from genotype to phenotype}

https://doi.org/10.1515/cclm-2018-1208

Received November 12, 2018; accepted February 22, 2019; previously published online June 7, 2019

Abstract: Loss of function mutations in the CYP24A1 gene, involved in vitamin $\mathrm{D}$ catabolism and in calcium homeostasis, are known to be the genetic drivers of both idiopathic infantile hypercalcemia (IIH) and adult renal stone disease. Recently, also defects in the SLC34A1 gene, encoding for the renal sodium-phosphate transporter $\mathrm{NaPi}$-IIa, were associated with the disease. IIH typically affects infants and pediatric patients with a syndrome characterized by severe hypercalcemia, hypercalciuria, suppressed parathyroid hormone level and nephrolithiasis. In SLC34A1 mutated carriers, hypophosphatemia is also a typical biochemical tract. IIH may also persist undiagnosed into adulthood, causing an increased risk of nephrocalcinosis and renal complication. To note, a clinical heterogeneity characterizes IIH manifestation, principally due to the controversial gene-dose effect and, to the strong influence of environmental factors. The present review is aimed to provide an overview of the current molecular findings on the IIH disorder, giving a comprehensive description of the association between genotype and biochemical and clinical phenotype of the affected patients. We also underline that patients may benefit from

\footnotetext{
*Corresponding author: Prof. Ettore Capoluongo, Laboratory of Molecular Diagnostics and Genomics, Teaching and Research Hospital “Fondazione Policlinico Agostino Gemelli" - IRCCS, Catholic University of the Sacred Heart, Rome, Italy, E-mail: ettoredomenico.capoluongo@policlinicogemelli.it Elisa De Paolis, Maria De Bonis and Angelo Minucci: Laboratory of Molecular Diagnostics and Genomics, Teaching and Research Hospital “Fondazione Policlinico Agostino Gemelli” - IRCCS, Catholic University of the Sacred Heart, Rome, Italy. https://orcid.org/0000-0002-0780-0153 (E. De Paolis) Giovanni Luca Scaglione: Laboratory of Molecular Oncology, "Fondazione Giovanni Paolo II", Catholic University of Sacred Heart, Campobasso, Italy
}

genetic testing into a targeted diagnostic and therapeutic workflow.

Keywords: calcium; CYP24A1; idiopathic infantile hypercalcemia; phosphate; SLC34A1; vitamin D.

\section{Introduction}

The idiopathic infantile hypercalcemia (IIH) is a rare genetic cause of nephrolithiasis (NL) typically occurring in pediatric subjects with an estimated incidence of 1:33,000 live birth [1]. The etiology has remained undefined for a long time, until the inactivation of cytochrome P450 family 24 subfamily A member 1 (CYP24A1) was identified as the molecular basis [2]. Recently, also the loss of function genetic variants in the solute carrier family 34 member 1 (SLC34A1) gene, coding for the NaPiIIa cotransporter, were ascertained as an additional pathogenic mechanism [3], widening the batch of the IIH genetic cause. The molecular heterogeneity allows the differentiation of two IIH forms, the IIH type 1 (OMIM 143880) caused by CYP24A1 genetic variants and the IIH type 2 (OMIM 616963) associated with SLC34A1 impairments. Both genetic defects lead to a shared pathological phenotype characterized by inappropriate increment of vitamin D active metabolites with persistent hypercalcemia, hypercalciuria and suppressed parathyroid hormone (PTH) [4]. These typical biochemical alterations lead to abnormal renal calcium (Ca) depositions with the consequent higher risk of $\mathrm{NL}$ and nephrocalcinosis (NC). SLC34A1 genetic variants carriers also manifest hypophosphatemia and renal phosphate $(\mathrm{Pi})$ wasting. Despite the term "infantile", the disease is not only confined to infancy as it may also affect subjects in later childhood and adulthood.

The challenge related to the correct IIH diagnosis lies in several pitfalls. Particularly, the peculiar 
genotype-phenotype relationship along with the strong influence of microenvironment triggering factors lead to a pattern of different clinical manifestations. Moreover, SLC34A1 pathogenic variants are associated with overlapping conditions in addition to $\mathrm{IIH}$, including hypophosphatemic nephrolithiasis with osteoporosis, type 1 (NPHLOP1, OMIM ID 612286) and Fanconi renotubular syndrome, type 2 (FRTS2, OMIM ID 613388).

Although IIH is a rare condition, the associated health effects can become severe if not promptly identified. Understanding of the IIH physiology is mandatory for the correct diagnosis and treatment. In this regard, the molecular evaluation has relevant and practical consequences in patients' care, giving the opportunity of targeted personalized therapies.

In the last decade, IIH assumed an emerging role among the genetic causes of pediatric-onset NL/NC, with an increment of case reports in the literature. The present paper provides an overview of the biological and pathological relevance of CYP24A1 and SLC34A1 defects in IIH. We focus on the genetic aspects of the disease, giving a comprehensive update on the mutations regarding both genes, with reference to computational models and in vitro mutagenesis studies supporting their functional characterization.

\section{Biological relevance of CYP24A1 and SLC34A1}

\section{CYP24A1 role in vitamin D catabolism}

CYP24A1 is a mitochondrial inner membrane $\mathrm{P} 450$ enzyme involved in the catabolism of vitamin D. It is expressed in many tissues including kidney, bone, skin and in the intestine [5, 6]. In the vitamin D metabolic pathway (Figure 1), the CYP24A1 enzyme catalyzes the conversion of the vitamin $\mathrm{D}$ biological active form $1,25(\mathrm{OH})_{2} \mathrm{D}_{3}$ into metabolites targeted for excretion. Particularly, the $1,25(\mathrm{OH})_{2} \mathrm{D}_{3}$ is inactivated by CYP24A1 through multiple hydroxylation steps at $\mathrm{C}-23$ or $\mathrm{C}-24$, resulting in the $1,25(\mathrm{OH})_{2} \mathrm{D}_{3}-26,23$ lactone and in the biliary catabolite

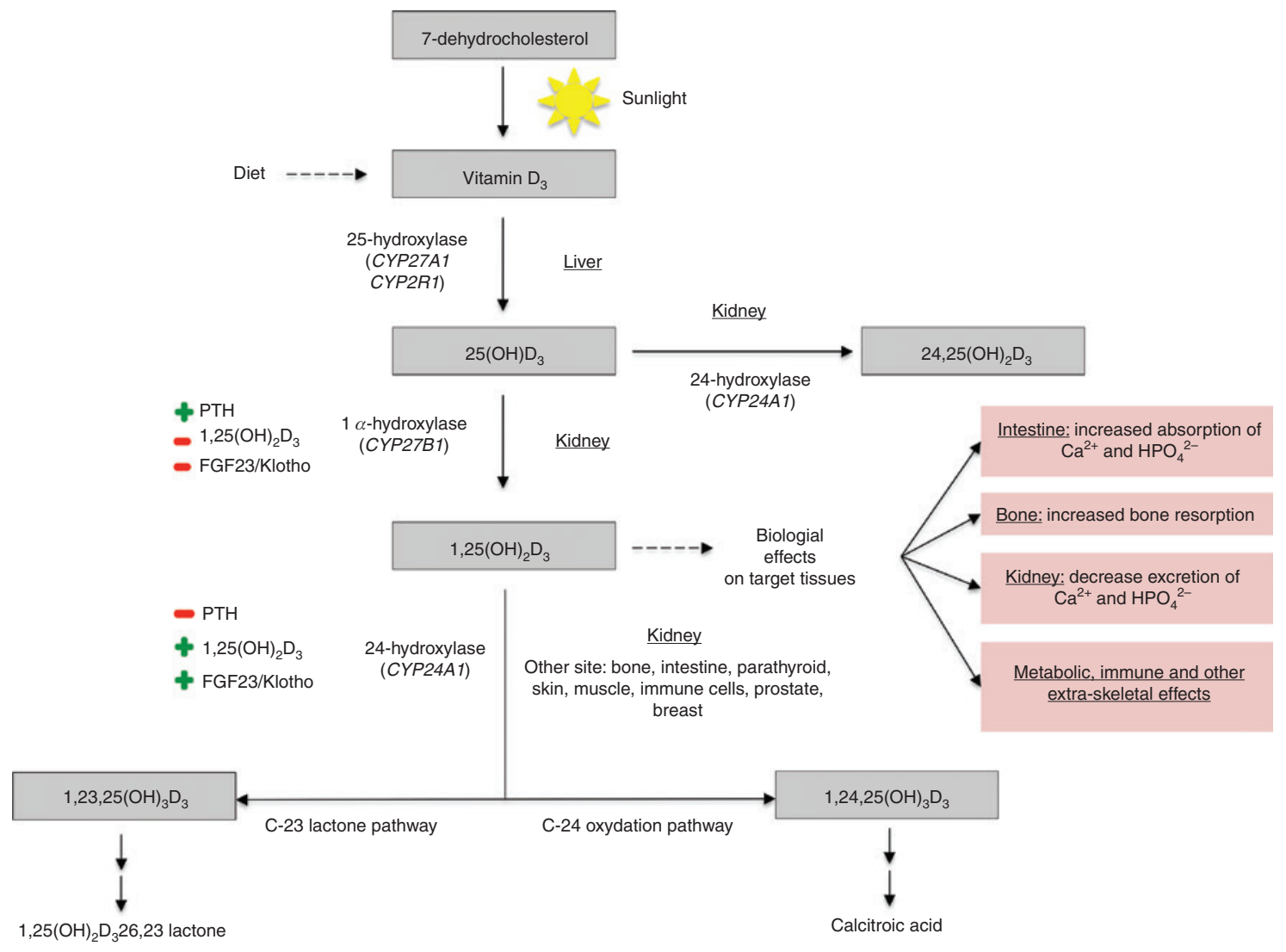

Figure 1: Overview of vitamin $D_{3}$ pathway and physiological role of CYP24A1.

Diagram shows the main steps of vitamin $\mathrm{D}_{3}$ metabolism, the enzymes involved in the metabolic pathway and the regulatory mechanisms. PTH, parathyroid hormone; FGF23, fibroblast grow factor-23. 
calcitroic acid, respectively. Also, the $25(\mathrm{OH}) \mathrm{D}_{3}$ metabolite is transformed by CYP24A1 into $24,25(\mathrm{OH}) \mathrm{D}_{3}$, which will be further converted to the water-soluble calcitroic acid $[7,8]$. The enzymatic activity of CYP24A1 is strongly regulated by several biochemical and genetic factors [9]. Firstly, the hypocalcemic condition results in the inhibition of Cyp24A1 expression through the destabilization and suppression of its mRNA turnover [10]. Several papers demonstrated how PTH downregulates CYP24A1 expression and enzymatic activity [11-13]. This regulatory effect results from both transcription and post-transcription modulation. Although PTH modestly increases CYP24A1 promoter activity [14], it also showed an important post-transcriptional effect on CYP24A1 mRNA stability, promoting its degradation [15-17]. To date, the specific PTH response elements in the CYP24A1 gene are unknown and many aspects of the regulatory mechanism remain to be elucidated [17]. Notably, the PTH inhibitory effect was not observed in extra-renal sites such as the intestine and epidermis [12, 18]. The opposite effect is mediated by the $1,25(\mathrm{OH})_{2} \mathrm{D}_{3}$ that regulates its own biosynthesis through a positive feedback on Cyp24a1, stimulating its gene expression [19]. In addition, the fibroblast growth factor 23 (FGF23), together with its co-receptor $\alpha$-Klotho, positively modulates CYP24A1 activity in response to hypercalcemia and hyperphosphatemia [10, 20]. The crucial role of CYP24A1 in the modulation of the $1,25(\mathrm{OH})_{2} \mathrm{D}_{3}$ intracellular concentration and half-life in the bloodstream, was widely investigated. A higher degree of impairment of $1,25(\mathrm{OH})_{2} \mathrm{D}_{3}$ and $25(\mathrm{OH}) \mathrm{D}_{3}$ pharmacokinetics was observed in Cyp24a1-null mice compared to heterozygotes Cyp24a1 ${ }^{+/-}$[5]. Particularly, loss of function mutations in the CYP24A1 gene result in the increase of both $25(\mathrm{OH}) \mathrm{D}_{3}$ and $1,25(\mathrm{OH})_{2} \mathrm{D}_{3}$ levels which enhance intestinal $\mathrm{Ca}$ absorption and bone reabsorption [21]. The consequent hypercalcemic phenotype is the major cause of IIH manifestations.

\section{SLC34A1 (NaPi-Ila) role in renal phosphate handling}

Body Pi levels are under a strong physiological control mediated by the intestinal uptake, the renal reabsorption/ excretion and the exchange between extracellular sites and bones [22]. In the human kidney, free-Pi is filtered in the glomerulus and subsequently reabsorbed in the range of $65 \%-70 \%$ along the nephron [23]. A group of transmembrane $\mathrm{Na}^{+}$-coupled Pi cotransporters (NaPi-II), belonging to the solute carrier family 34 (SLC34), plays a central role in Pi handling [24]. Particularly, the isoform
NaPi-IIa (SLC34A1), expressed in the apical brush-border membrane (BBM) of the proximal tube [25], plays the most important role in the conservation of filtered $\mathrm{Pi}$ from primary urine, being responsible for $60 \%-70 \%$ of this in rats and mice [23,26]. The renal Pi handling via NaPi-IIa is influenced by several regulatory mechanisms that adjust BBM cotransports expression to the physiological need [22, 27, 28]. Particularly, the NaPi-IIa is regulated by vitamin D and PTH, together with other non-classical mineral metabolism components, such as the phosphatonins FGF23 and $\alpha$-klotho [29]. 1,25(OH) ${ }_{2} \mathrm{D}_{3}$ modulates both intestinal and renal Pi handling upregulating the NaPi-II cotransporters expression [23, 30, 31]. However, conflicting findings surrounding the direct effects of $1,25(\mathrm{OH})_{2} \mathrm{D}_{3}$ on Pi renal reabsorption have been reported [30, 32]. In vitamin D-deficient rats, the expression of NaPi-IIa cotransporter in juxtamedullary kidney cortex is significantly reduced and the administration of $1,25(\mathrm{OH})_{2} \mathrm{D}_{3}$ leads to normalization of NaPi-IIa expression and Pi uptake [30]. Also, in vitamin D receptor (Vdr)-null growing mice, Kaneko et al. observed a reduced renal Pi reabsorption with hypophosphatemia [33]. However, in this mouse model, a rescue diet completely normalized the expression of the renal Slc34a1 cotransporter together with the renal Pi reabsorption. This observation suggested that the alteration in Pi homeostasis observed in Vdr-null mice could not be directly related to the loss of vitamin D signaling. On the contrary, it could be the result of high PTH levels [33]. PTH promotes Pi renal excretion stimulating NaPi-IIa endocytosis from the apical membrane of the proximal tubule cells $[28,34]$. Like PTH, a high Pi diet also induces down-regulation of NaPi-IIa probably through a similar mechanism, even if this process was not deeply studied [35]. FGF23 is an additional regulatory element, which is produced within the bone in response to high vitamin D levels. In the kidney, FGF23 acts as phosphaturic factor inducing the downregulation of NaPi-IIa expression, in cooperation with the cofactor $\alpha$-klotho [22, 34]. Taking into account the complex cross-talk between the regulatory mechanisms of both Ca and Pi metabolism pathways, it is not surprising as the impairment of NaPi-Ila activity also leads to relevant consequences in Ca mineral balance. IIH infants carrying SLC34A1 inactivating mutations show a persistent condition of hypophosphatemia. Experimental data from animal studies demonstrated that FGF23 serum concentration directly varies with Pi serum concentration [36]. This correlation was also observed in human studies, although with some controversial results [27]. The hypophosphatemia itself, together with the suppressed concentration of FGF23, leads to the stimulation 
of CYP27B1 and simultaneous inhibition of CYP24A1 activity $[36,37]$. The resulting increment of $1,25(\mathrm{OH})_{2} \mathrm{D}_{3}$ levels leads to the hypercalcemic phenotype [3].

\section{Pathological relevance of CYP24A1 and SLC34A1 defects}

The first clinical reports of IIH were described in the 1950 s in the UK, when an epidemic peak of IIH was observed after the introduction of an increased prophylactic dose of vitamin D in infant formula and milk products [38]. After halving of the vitamin D dose, a concomitant decrease of the incidence was observed. In the 1970s, a similar phenomenon was reported in Poland [39] and in Eastern Germany [40]. In the same years, a genetic basis related to defective mechanisms of vitamin D inactivation was proposed as one of the possible cause of the disease [41-43]. Sixty years later, inactivating mutations accounted in homozygosis or in compound heterozygosis within the CYP24A1 gene were identified as the specific IIH genetic cause [2]. Moreover, in 2016 Schlingmann and coworkers described a subgroup of IIH patients without CYP24A1 mutations showing idiopathic renal Pi wasting together with the other IIH typical biochemical traits. In these patients, the disturbances observed in Ca homeostasis resulted as the consequence of the primary defect in Pi metabolism caused by SLC34A1 genetic variants. In this cohort, as opposite to CYP24A1-mutated subjects, the clinical and laboratory findings also persisted after the discontinuation of vitamin $\mathrm{D}$ prophylaxis, while a rapid response to Pi supplementation was registered [3]. These findings supporting the role of SLC34A1 as alternative genetic driver of $\mathrm{IIH}$, emphasize the molecular heterogeneity of the disease and open an additional way to increase the number of patients in which a definitive molecular diagnosis could be reached.

$\mathrm{IIH}$ is a rare condition mainly characterized by severe hypercalcemia with hypercalciuria, suppressed PTH and deposits of $\mathrm{Ca}$ in the kidney (NL/NC), with a predisposition to renal complications. Typical manifestations of IIH include also polyuria with dehydration, vomiting and constipation [2]. Anorexia and weight loss are reported, together with the difficulty in healthy eating acquisition [44]. Affected babies could also exhibit muscular hypotonia and lethargy, with an inability to grow [45]. Delay in the development of mental and movement abilities were also reported [46]. IIH is not restricted to infancy and its clinical manifestations may appear later into adult life. In this case, the sub-clinical long-term hypercalcemia/ hypercalciuria causes kidney stones disease in adulthood with a more severe damage of kidney function [47-49]. Affected adults may also develop extra-renal manifestations, including Ca deposits in the joints [50] and in the cornea, a low bone mineral density and osteoporosis, principally related to the increase of the osteoclast activity [51-53].

\section{CYP24A1 pathological role: the peculiar genotype-phenotype correlation}

The association between CYP24A1 defects and Ca homeostasis impairment with hypercalcemia and kidney stones is widely supported by experimental studies. The pathological phenotype observed in mouse model with homozygotes loss of function mutations in Cyp24a1 includes hypercalciuria, abnormal kidney morphology and dilated renal tubules, together with bone mineralization defects [19]. In a recent paper, Jing et al. defined CYP24A1 as a novel candidate gene for kidney disease pathogenesis [54]. Moreover, this enzyme deficiency is estimated to characterize $4 \%-20 \%$ of all Ca kidney stone forming patients [53]. IIH caused by homozygous or compound heterozygous variants in the CYP24A1 gene shows an autosomal recessive inheritance. However, high variability in the disease expression emerges as a result of genetic and environmental cross-talk. Clinical and biochemical phenotypes are featured by a gene-dose effect: subjects with bi-allelic genetic variants show a more severe phenotype compared to mono-allelic carriers, with the latter being asymptomatic or borderline $[55,56]$. Moreover, in the heterozygote individuals, the chronic and latent abnormalities of vitamin D metabolism seems more likely to predispose to long-term clinical events such as renal stones [57]. In some cases, this clinical outcome variability was suggestive of a dominant inheritance with reduced penetrance [58]. In addition, differences in the effects of mono-allelic haplo-insufficiency were also observed with respect to the age of onset: heterozygote infants typically show higher $1,25(\mathrm{OH})_{2} \mathrm{D}_{3}$ and lower PTH levels than adults, suggesting that the prematurity in the renal function could influence the tolerance to vitamin D [59]. In addition to the genetic background, the IIH manifestations and the phenotypic differences between infants and adult carriers rely on many environmental factors like diet, lifestyle, vitamin D intake and activity of the other vitamin D metabolism enzymes [60]. Sunlight exposure and vitamin D supplementation therapy were identified as the two main environmental triggering factors [61, 62]. In particular, Schlingmann et al. described CYP24A1-mutated asymptomatic children 
who rapidly manifested the IIH phenotype only after the administration of a high dose of prophylactic vitamin D [2]. The higher intake of Ca and vitamin D is a common practice not only in infant diets but also during pregnancy and lactation, in order to preserve maternal, fetal and child health. During the gestational period, adaptive mechanisms naturally occur in order to meet fetal needs $[63,64]$. In women carriers of CYP24A1 defects, these physiological changes, coupled with the pharmacological treatment, could unmask an underlying IIH phenotype and lead to gestational maternal hypercalcemia, with possible hypertension and fetal demise [62, 65]. With regard to the exposure to sunlight, a significant seasonal variation was observed in IIH patients with a trend to higher calcemia and calciuria, as well as lower PTH levels, from April to September (at greater exposure) as compared to the winter months [52]. Taken together, these findings support the conclusion that the dose and as well as the type of vitamin $\mathrm{D}$ administration may drastically modulate adverse effects. Thus, CYP24A1-deficient subjects are recommended to carefully observe their vitamin $\mathrm{D}$ intake or to completely restrict its intake. Consequently, the IIH diagnosis via the identification of CYP24A1 mutations carriers is also useful for the appropriate vitamin D supplementation scheme. Both protection and/or mild exposure to the sun are also suggested to prevent the worsening of clinical and biochemical manifestations [52].

\section{SLC34A1 pathological role: heterogeneity of clinical phenotype}

The pathophysiology underlying $\mathrm{NC} / \mathrm{NL}$ in carriers of SLC34A1 genetic variants is based on the primary loss of Pi in the urine. The Pi wasting and the suppression of FGF23 lead to an inappropriate $1,25(\mathrm{OH})_{2} \mathrm{D}_{3}$ synthesis via the inhibition of CYP24A1 catabolic activity and, on the other hand, via the stimulation of CYP27B1. The consequent status of hypercalcemia, hypercalciuria and hyperphosphaturia promotes the formation of Ca-Pi crystals and renal calcifications [66]. Mutations in the SLC34A1 gene were widely associated with kidney stones phenotypes. In particular, SLC34A1 was annotated among the relevant genes for idiopathic Ca stones in several human GWASs and replicated candidate-gene studies [67, 68]. Extensive studies proved the pathological role of both complete deletion and loss of function genetic variants in the SLC34A1 gene [69, 70]. Homozygous knockout (KO) mice exhibit hypophosphatemia and renal Pi wasting with an adaptive increase of the serum $1,25(\mathrm{OH})_{2} \mathrm{D}_{3}$ and associated hypercalcemia and hypercalciuria, together with renal $\mathrm{Ca} / \mathrm{Pi}$ depositions with an apatitic mineral phase [71, 72]. Additionally, a phenotype with hypophosphatemia, hypercalcemia and NL was observed in mice carriers of point mutations inactivating the Slc34a1 gene [73]. As described for CYP24A1, autosomal recessive inheritance is associated with SLC34A1 genetic variants, with a phenotype variability related to mono- and bi-allelic defects. Heterozygotes Slc34a1 $1^{+-}$mice are partially able to maintain normal serum Pi levels with a modest increase in urinary Pi excretion. These findings suggest that kidneys of Slc34a1 ${ }^{+/}$ mice are able to react properly to the increase of renal Pi loss and, to the resulting modification of Pi homeostasis [69]. Schlingmann et al. evaluated Slc34a1 KO mice fed with diets characterized by variable content of vitamin D and Pi. A low Pi diet led to severe hypophosphatemia only in the KO mice whereas wild type (WT) animals remained normophosphatemic. Furthermore, the high vitamin D diet resulted in its overload that is not compensated in the $\mathrm{KO}$ animals. In a lowPi/highD diet, representative of infants with breast milk alimentation and vitamin D fortification, only the KO mice showed the IIH manifestations due to their lack of FGF23 regulatory activity. Furthermore, avoiding the vitamin $\mathrm{D}$ intake does not lead to the normalization of laboratory findings as it was obtained only after the Pi load $[3,70]$. As discussed before, the association of IIH with SLC34A1 defects was discovered recently [3]. Until then, genetic variants in this gene were known to give several clinical phenotypes that overlap with IIH symptoms mainly related to hypophosphatemia [74]. The first condition associated with heterozygote genetic variants in the SLC34A1 gene is NPHLOP1. Common manifestations are persistent idiopathic hypophosphatemia with Pi wasting, hypercalciuria, bone demineralization and kidney stones [75]. However, this autosomal dominant form was not confirmed in subsequent studies [76-78]. An autosomal recessive form related to genetic variants in the SLC34A1 gene is FRTS2, described for the first time in Israeli-Arab patients [79]. Particularly, Tieder et al. described two adolescent siblings with severe hypophosphatemic rickets and osteopenia, severe hypercalciuria without renal tubular acidosis and significantly elevated serum levels of $1,25(\mathrm{OH})_{2} \mathrm{D}_{3}$. Clinical reassessment of these patients after two decades also revealed proximal tubulopathy, normocalcemia, normocalciuria, renal $\mathrm{Pi}$ wasting with hypophosphatemic bone disease and stage II-III chronic kidney disease (CKD) [78]. However, the occurrence of FRTS2 in the molecular context of SLC34A1 genetic variants is unclear [22]. Despite that there is phenotypic similarity between humans FRTS2 and mice models with $\mathrm{NaPi}-\mathrm{IIa}$ disruption, there are several unexplained differences between the two species, including 
the absence of: (a) clear FRTS2 phenotype with classic rickets in the affected mice and (b) renal calcification in the affected humans [78]. To date, no additional patients with NaPi-IIa disruption and FRTS2 have been reported. Additionally, several case reports described IIH with NC in patients affected by Sotos syndrome. This condition is caused by a heterozygous microdeletion on chromosome 5 q35 removing both the nuclear receptor binding SET domain protein 1 (NSD1) and the SLC34A1 genes. Together with the typical manifestations of Sotos syndrome related to the NSD1 gene deletion, the mineral disorders observed were linked to NaPi-IIa haploinsufficiency [80].

Due to the phenotype variability in homozygotes carriers and to the debate regarding the impact of heterozygotes genetic variants, the clinical effects of NaPi-IIa impairments in humans show some controversial aspects. To date, pathogenic alleles of SLC34A1 seem to contribute to both autosomal dominant and autosomal recessive renal stone disease, suggesting the importance of other factors that can influence the clinical features of the disease [74].

\section{Genetic variant spectrum of CYP24A1 and SLC34A1}

All the genetic variants accounted in CYP24A1 and SLC34A1 genes and associated with IIH are shown in Tables 1 and 2, respectively. The present variants are reported and described in the following databases: (1) Leiden Open Variant (http://www.lovd.nl/3.0/home) [95]; (2) Human Gene Mutation (http://www.hgmd.cf.ac.uk/ac/ index.php) [96] and (3) ClinVar (https://www.ncbi.nlm. nih.gov/clinvar/) [97]. Through the MEDLINE consultation, all potentially relevant primary papers (last released Dec 2018) were examined. Furthermore, the reference list was double checked for additional publications. Recommendations of the Human Genome Variation Society [98] were followed for the description of variants and a checking of the correct nomenclature was performed using Mutalyzer 2.0.26 tool (http://lovd.nl/mutalyzer/) [99]. In particular, for each genetic variant we herein provide details including:

1. The exon/intron involved, followed by the genomic and coding position (GRCh38/hg19) based on the reference sequences NG_008334.1 and NM_000782.4 for the CYP24A1 gene and NG_016223.1 and NM_003052.4 for the SLC34A1 gene. Nucleotide numbering at coding sequence starts with c.1 at the "A" of the "ATG" start codon that corresponds to the genomic position g.5399 for CYP24A1 and g.6312 for SLC34A1, according to the mentioned reference sequences.

2. The type of genetic variant, reported as: missense, nonsense, frameshift, in-frame deletion/duplication, splice site mutation, large deletion.

3. The effect predicted at the protein level is reported as referred to the CYP24A1 NP_000773.2 and SLC34A1 NP_003043.3 accession numbers, with the indication of the predicted protein domain involved, according to the published structural information [100, 101].

4. Annotations details for the Reference SNP (rs) ID number obtained from Database of Single Nucleotide Polymorphism (dbSNP) (https://www.ncbi.nlm.nih. gov/projects/SNP/) [102].

5. Data supporting the pathological significance of the mutations obtained from the published functional studies based on in silico prediction algorithms and/ or in vitro testing. For those variants with unknown functional impact, we performed a prediction of the pathogenicity using the following in silico tools: PolyPhen-2 (http://genetics.bwh.harvard.edu/pph2/) [103], MutationTaster (http://www.mutationtaster. org/) [104] and SIFT algorithm (http://sift.jcvi.org/) [105]. The 3' and 5' splice sites analysis was achieved using Human Splicing Finder v3.1 tool (http://www. umd.be/HSF3/) [106].

A comprehensive description of each variant encompassing all molecular, biochemical and clinical details is available for both CYP24A1 and SLC34A1 genes in Supplementary Tables 1 and 2, respectively. Additionally, graphical linear maps of the pathogenic variants spanning the genes are depicted in Figure 2. Notably, the IIH case reports with definitive genetic diagnosis were more frequently associated with CYP24A1 defects compared to SLC34A1. The limited number of pathogenic variants reported is mainly due to the very recent discovery of the gene-disease association, since the first evidence was published in 2011 for CYP24A1 and 2016 for SLC34A1 [2, 3]. Additionally, the IIH can be considered an under-diagnosed condition due to the high variability of its clinical manifestations together with the lack of genetic analysis routinely performed.

\section{CYP24A1 pathogenic variants in IIH}

A total of 41 pathogenic variants were identified for the CYP24A1 gene. The majority of the protein domains results as affected by one or more deleterious amino acid changes (Figure 3). Overall, the most common types of genetic variants are missense (23/41), followed by 


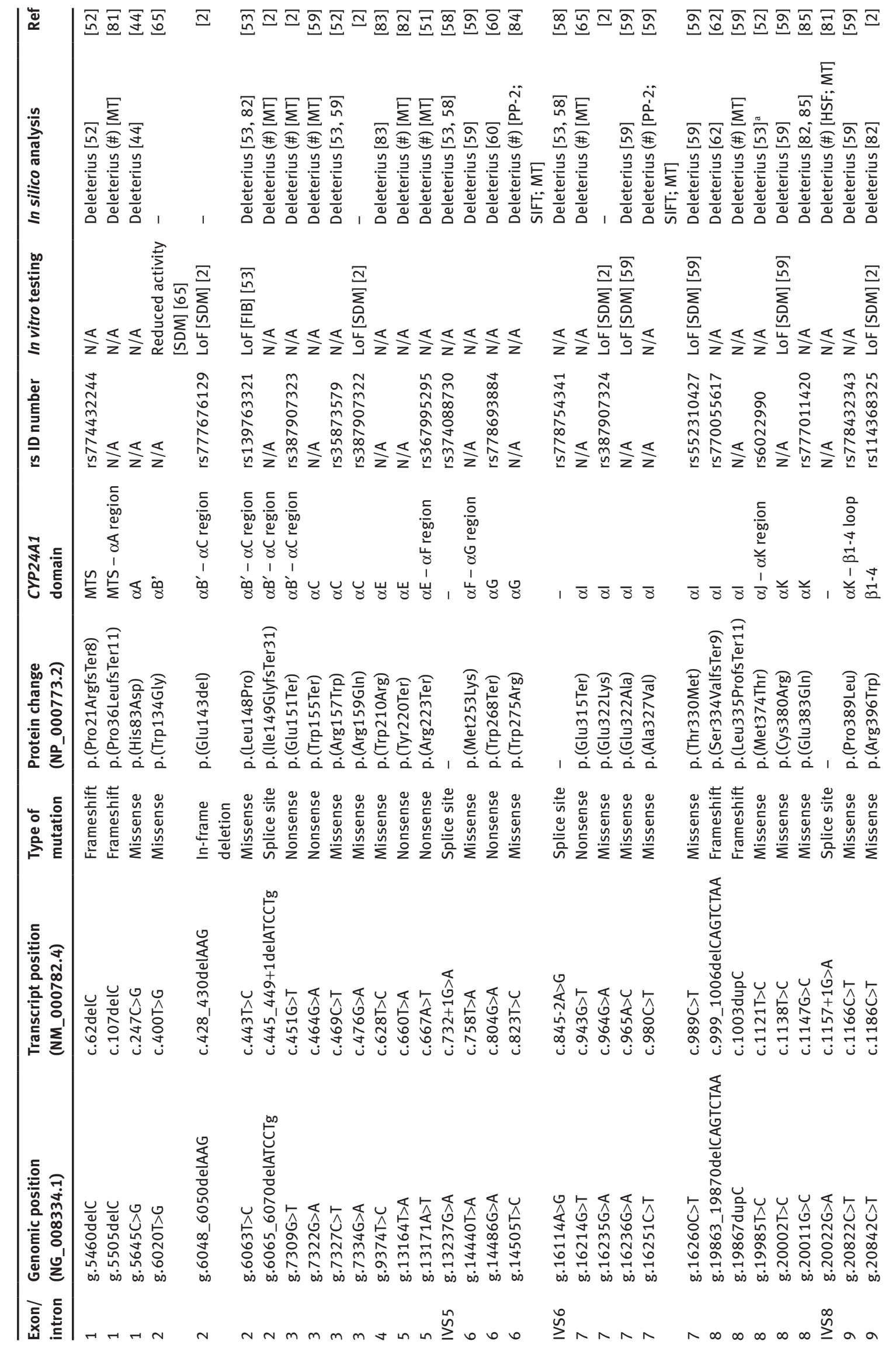




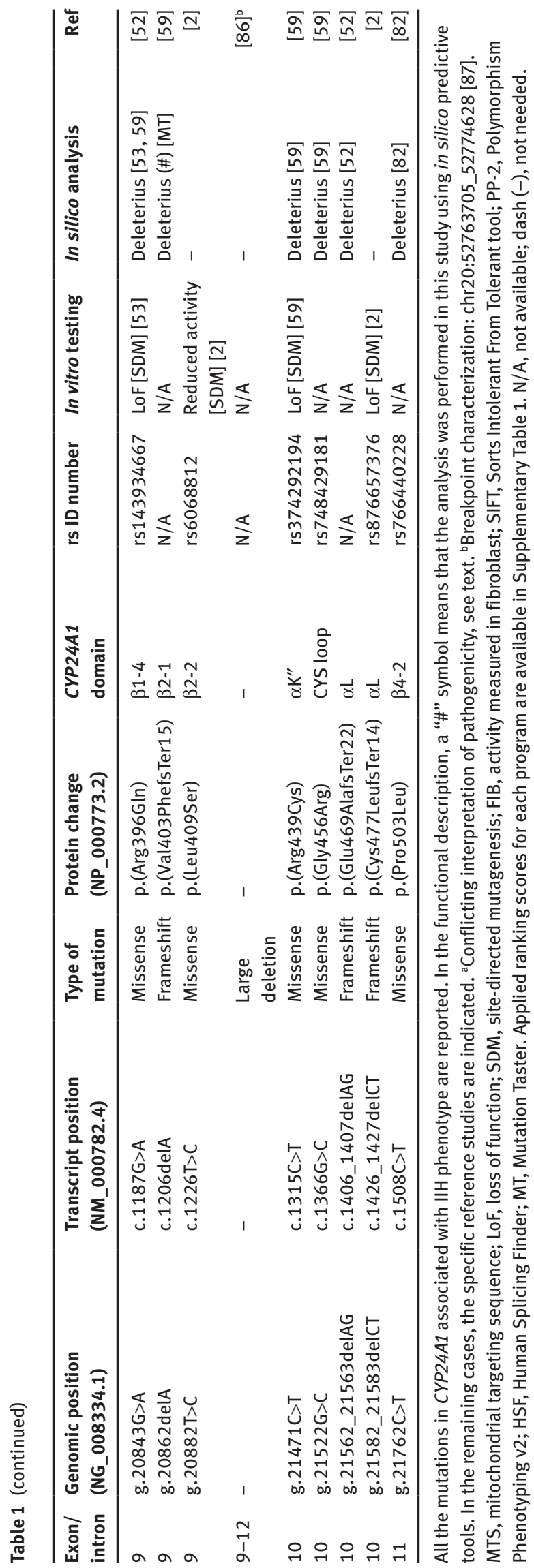

the frameshift and in-frame deletions (7/41), nonsense (5/41), splice site changes (4/41), frameshift duplications (1/41) and large deletions (1/41). Relevant details about the effect of the genetic variations on the enzyme activity were obtained through in vitro site-direct mutagenesis (SDM) analysis and computational model via web-based tools [109]. Genetic variants within CYP24A1 can affect the enzyme structure and catalytic activity, through several mechanisms, including: (a) the alteration of both the substrate (e.g. p.Leu409Ser; p.Leu148Pro) and the heme (e.g. p.Arg159Gln; p.Arg396Trp; p.Ser334ValfsTer9) binding; (b) the substrate access (e.g. p.Glu143del) and the product egress; (c) the disruption of ferredoxin cofactor binding (e.g. p.Glu383Gln) and d) the protein folding (e.g. p.Glu322Lys) [2, 53, 82, 110]. In particular, the c.476G $>A$ (p.Arg159Gln) and the c.1186C $>$ T (p.Arg396Trp) variants influence the heme binding site due to the destruction of the hydrogen bond between the heme propionate group and the arginine residues [110]. Consequently, both the protein structure stability and the catalytic proprieties are drastically impaired [109]. Instead, the binding of the $1,25(\mathrm{OH})_{2} \mathrm{D}_{3}$ substrate is influenced by the c.1226T $>C$ (p.Leu409Ser) mutation, causing the decrease of enzymatic activity [53]. Notably, the leucine 409 does not directly contribute to the substrate-binding pocket or to the substrate access channel and both the WT Leu and the alternative serine residues have similar volumes [111]. The functional effect of this amino acid substitution is probably related to the conformational change of an $\alpha$-helix into a $\beta$-turn [109]. The amino acid residue Leu148 directly interacts with the biochemical substrate where the substitution c.443T $>C$ (p.Leu148Pro) leads to a decrease of the enzyme activity of about $25 \%-50 \%$ [53]. Computational analysis demonstrated that this genetic variant determines the change of a small coil with an impaired protein stability [109]. The c.964G $>$ A (p.Glu322Lys) substitution abolishes an important hydrogen bond between the I-helix and the $\mathrm{B}^{\prime} / \mathrm{C}$ loop backbone, altering their orientation and affecting the correct protein folding and stability [112]. Moreover, Masuda et al. demonstrated that the substitutions of the residues Trp134 and Leu148, together with Met246 and Ile131, affect the substrate orientation into the enzyme channel and lead to the decrease of the enzymatic activity [113]. Among the CYP24A1 genetic variants reported in the Table 1, the small deletion c.428_430delAAG (p.Glu143del) is one of the most common in IIH patients, being that it has been described in many case reports $[2,48,51,53,59,60,65$, $82,85,112]$. This variant represents a mutational hot spot of the CYP24A1 gene, together with the c.1226T $>C$ 


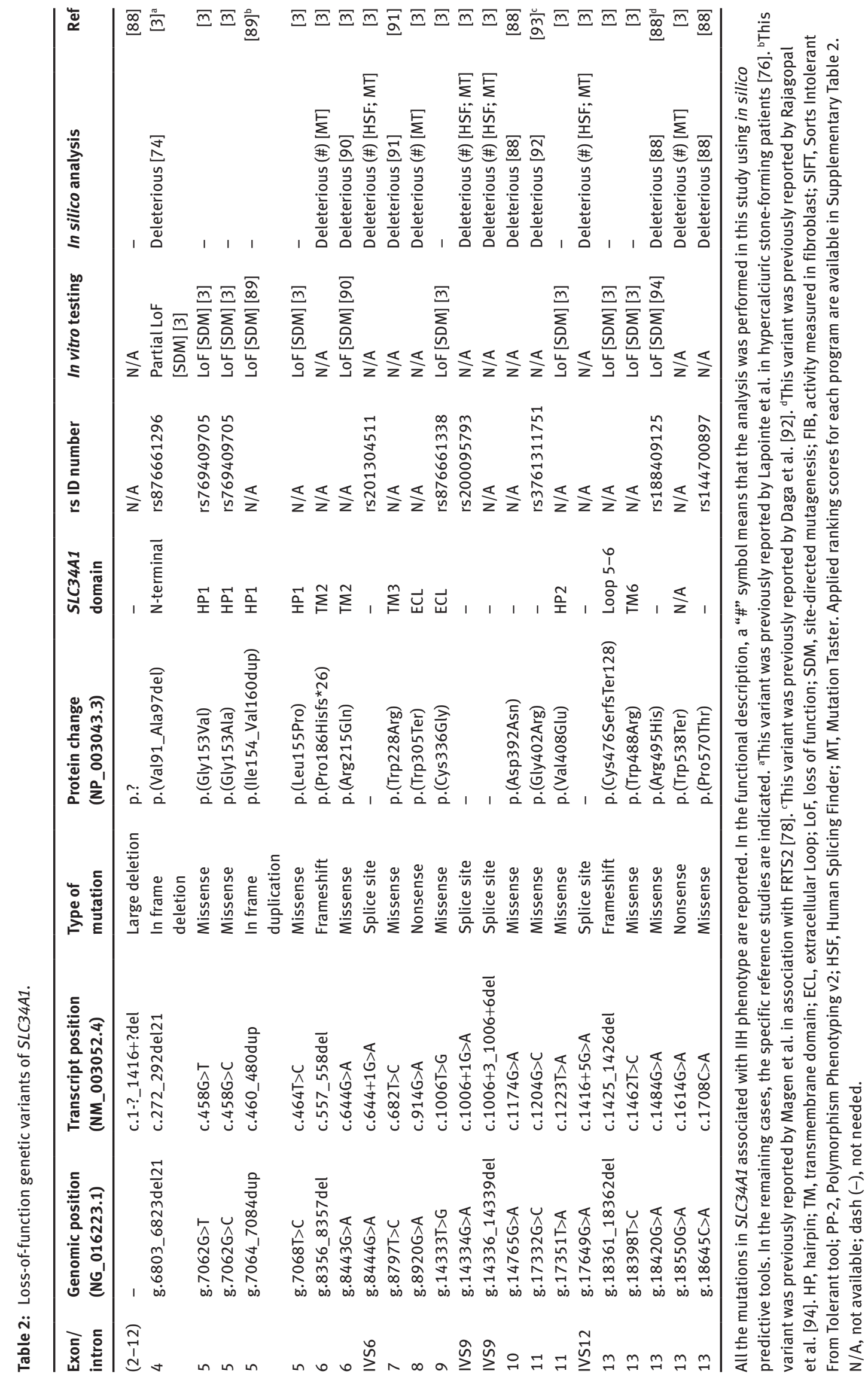


A
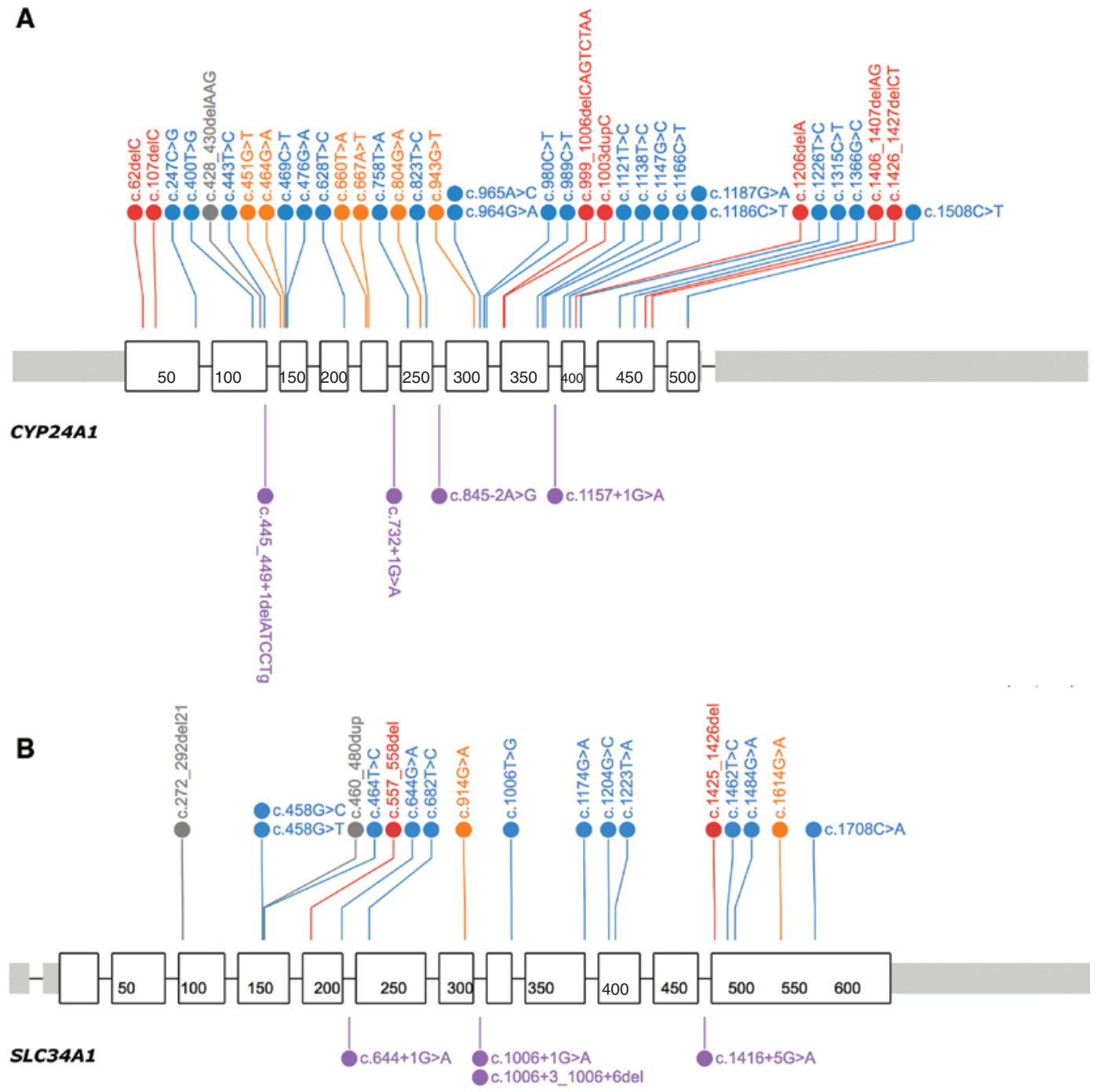

Figure 2: Distribution of IIH-causing mutations in the context of CYP24A1 and SLC34A1 gene structures.

For both CYP24A1 (A) and SLC34A1 (B), the Figure shows the linear maps of the gene and the location of the pathogenic mutations. Each rectangle represents a single exon, whereas the horizontal lines represent intronic regions. Missense (blue), non-sense (orange), frameshift (red) and in-frame indels (gray) are shown on the top, whereas splice-site mutations (purple) are shown below. The figures were obtained by custom tracks feature of the web tool ProteinPaint [107].

(p.Leu409Ser) in the French population [59] and the c.1186C > T (p.Arg396Trp) in Italians [82]. The deletion of this highly conserved amino acid has a great impact on the enzyme function, destabilizing the $\mathrm{B}^{\prime} / \mathrm{C}$ loop and causing a reorientation of the $\mathrm{B}^{\prime}$-helix. The consequent impairment of the substrate binding cavity shape causes a drastic reduction of enzyme activity in vitro [2]. Also in vivo biochemical functional data provide strong evidence about the impact of this mutation in the IIH development [114]. Interestingly, the genetic variant c.823T $>C$ (p.Trp275Arg) was retrospectively identified in a patient presenting with hypercalcemia, high 1,25(OH)D level and elevated $25(\mathrm{OH}) \mathrm{D} / 24,25(\mathrm{OH})_{2} \mathrm{D}$ ratio, providing the impairment of CYP24A1 catabolic activity [84]. The c.1121T >C ( .Met374Thr) variant has conflicting interpretations. Molin et al. described this variant as a polymorphism rather than a mutation, considering its unusually high MAF ( $=0.0083)$ and the corresponding measurable CYP24A1 in vitro activity [59]. Despite these findings, the variant is described as causative of IIH in compound heterozygosis with the c.469C>T (p.Arg157Trp) [52] and as a pathogenic by in silico predictive algorithms [53, 59]. Finally, we highlight as the CYP24A1 missense variant NM_000782.4: c.735G $>$ A (NP_000773.2: p.Met245Ile) is not reported in the Table 1 due to its lack of association with a clear IIH phenotype. This variant 


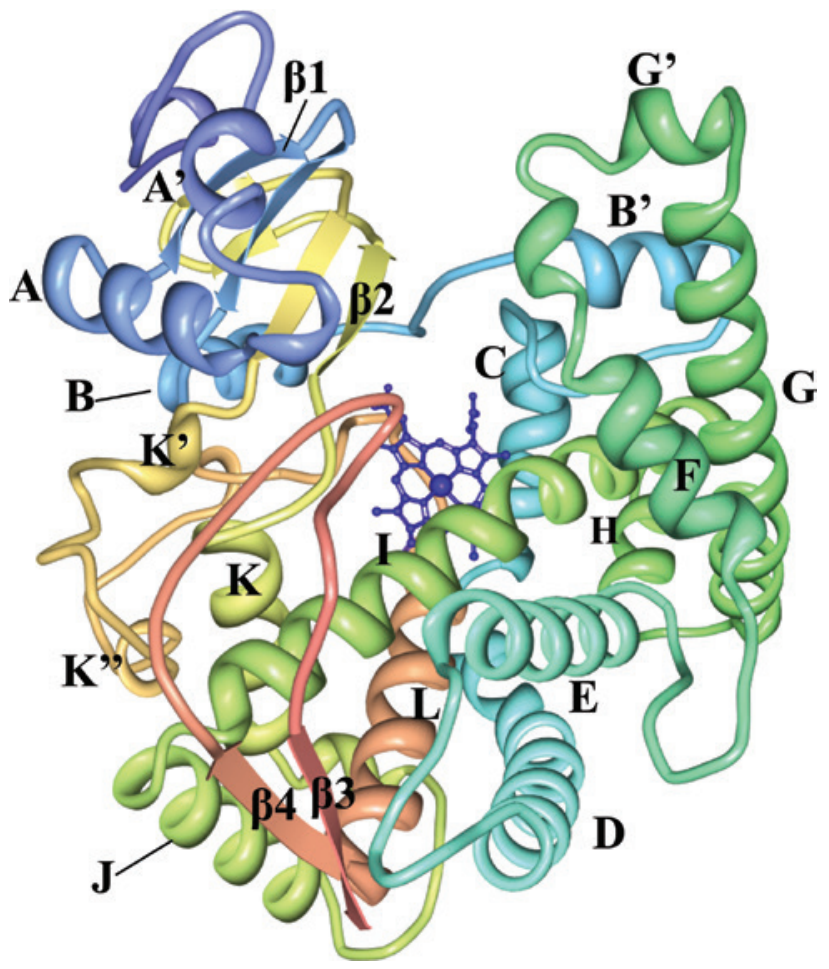

Figure 3: The crystal structure of rat CYP24A1.

Figure shows the crystal structure of rat CYP24A1 (PDB ID: 3K9V) [100] created with Protein Workshop 4.2.0 tool [108]. The CYP24A1 structure are colored according to a chain color ramp from $\mathrm{N}$-terminal (purple) to C-terminal (red).

within exon 6 of the CYP24A1 gene involves the highly conserved amino acid residue occurring in the polar region of the $\mathrm{F}$ helix: it is annotated with a very low MAF ( $=0.00045)$. Gigante et al. detected this genetic variant in homozygote status in an asymptomatic child during genetic screening [82]. This Italian proband (14 years old) showed biochemical findings within the normal range: nevertheless, the putative role of this variant in a late onset manifestation of the disease or in a latent phenotype was hypothesized. The authors also underlined that this asymptomatic child never received vitamin D supplementation. As reported by other authors, vitamin D supplementation can unmask a latent IIH condition due to the incomplete penetrance of genetic background [2]. Functional in silico analysis of p.Met245Ile proved a loss of enzymatic activity [82]. Additionally, the Met245 residue plays a relevant role in the catalysis, as evidenced by rat site-directed mutants that showed changes in binding affinity for the $1,25(\mathrm{OH})_{2} \mathrm{D}_{3}$ substrate along with an enhancement in the $25(\mathrm{OH}) \mathrm{D}_{3}$ ones [115]. However, definitive in vitro experiments for the specific p.Met245Ile variant are necessary to establish its biological significance.

\section{SLC34A1 pathogenic variants in IIH}

The spectrum of SLC34A1 genetic alterations in IIH comprises truncating as well as missense and splice site variants. According to the predicted NaPi-IIa structure model [101], most of these mutations appear to be located within transmembrane helices (TMs) domains. Several genetic changes were functionally tested in vitro, in heterologous expression systems, especially in Xenopus oocytes or in opossum kidney (OK) cells. As indicated in Table 1, altogether these experiments showed a reduced function of the mutated transporters, mainly due to trafficking defects. Interestingly, the small in-frame deletion c.272_292del21 (p.Val91_Ala97del) was reported for the first time by Lapointe et al. in hypercalciuric stone-forming patients. Despite the authors defined a lack of association between NaPi-IIa alterations and renal Pi handling, we would underline as only heterozygote patients were analyzed in this study [76]. The same variant was successively detected in compound heterozygosis with the c.1534C $>$ T (p.Arg512Cys) in an infant with kidney stones and primary hyperoxaluria [116]. Schlingmann et al. described the same variant in homozygote IIH carriers [3]. The pathological relevance of the c.272_292del21 (p.Val91_Ala97del) mutation was also supported by paper regarding an infant with Sotos syndrome and clinical IIH. This patient carried both the typical Sotos micro-deletion involving the SLC34A1 gene on one allele and the p.Val91 Ala97del variant on the other allele [80]. Particularly, the deletion is located in the N-terminus of the NaPi-IIa protein and probably its occurrence arose from a DNA strand slippage due to the pairing between homologous sequences of $5 \mathrm{bp}$ flanking the deleted region [76]. In vitro functional analysis of p.Val91_Ala97del mutant showed an unclear transport defect, with a Pi transport activity comparable with the WT [3] or lower than WT [76]. The transfection of EGFP-tagged into OK cells demonstrated a partial retention of the protein, with an expression in both the intracellular compartment and plasma membrane. However, all patients carrying this deletion presented with a clinical phenotype similar to those with other IIH pathogenic variants, proving its pathogenicity role in the disease occurrence. Additionally, the c.458G $>\mathrm{T} / \mathrm{C}$ (p.Gly153Val/Ala), c.464T>C (p.Leu155Pro), c.1006T $>\mathrm{G}$ (p.Cys336Gly), c.1223T $>A$ (p.Val408Glu), c.1425_1426del (p.Cys476Serfs $\left.{ }^{\star} 128\right)$ and c.1462T>C (p.Trp488Arg) variants were tested in vitro as loss of function mutations due to a disturbed trafficking and a complete intracellular retention of the NaPi-IIa [3]. The c.644G $>$ A (p.Arg215Gln) variant is predicted to be located in the first structural repeat unit (RU) of the protein, particularly the amino acid 
substitution affects a charged residue of the TM2 domain, altering the helical packing and/or the mobility of the transporter. Moreover, in vitro analysis confirmed a reduction of Pi intake transport of the mutated transporter and an impaired trafficking to the cellular membrane, with only a marginal effect on the transport behavior $[90,101]$. Recently, in a large study analysis of target tubulopathy genes, Ashton et al. described the c.1484G $>$ A (p.Arg495His) variant in an IIH patient [88]. This variant was previously identified in two Argentinean siblings (2 years old and 10 years old) with not persistent hypercalcemia, hypercalciuria, hypophosphatemia, low PTH level and NC [94]. This amino acid substitution resides in a domain essential for PTH-mediated endocytosis of NaPi-IIa and in vitro Pi uptake experiments showed a reduction in the amount of $\mathrm{Pi}$ transported [94]. The genetic variant c.1204G $>\mathrm{C}$ (p.Gly402Arg) was reported for the first time by Daga et al. in an Egyptian patient affected by nephrocalcinosis [92]. In a recent paper, the same variant was described in a case report of prenatal hyperechogenic kidneys corresponding to postnatal nephrocalcinosis [93]. The c.460_480dup (p.Ile154_Val160dup) variant was reported by Demir et al. in two infants of Israeli and Turkish descent, presenting with IIH and early-onset partial proximal tubular impairment [89]. This SLC34A1 genetic variant was previously reported in association with FRTS2 and CKD in the same families' older members [78]. Either the intra-familiar phenotypic heterogeneity or an age-dependent disease progression, observed between homozygotes adult and infant carriers, is still pending further investigations. Of interest, several SLC34A1 mutations are annotated with a high minor allele frequency (Supplementary Table 2). It is currently unknown why SLC34A1 mutations have accumulated such a high frequency in the general population [117]. This finding reinforces the debate surrounding SLC34A1 defects and suggests the strong influence of environment as well as other physiological factors in the development of a clear pathogenic phenotype.

\section{SLC34A1 pathogenic variants in other phenotypes}

Due to the mentioned controversial aspects related to the clinical heterogeneity that characterizes NaPi-IIa defects, another two different phenotypes, in addition to IIH, can be mentioned. An overview of the published pathological variants accounted in the SLC34A1 gene associated with these additional phenotypes is reported in Table 3. In the first clinical description of the NPHLOP1 condition, Priè et al. evaluated two heterozygote children, carrying the missense variants c.142_143delGCinsTT (p.Ala48Phe) and c.439G $>A$ (p.Val147Met). Functional analysis of these protein alterations confirmed an impaired NaPI-IIa transport activity [75]. On the other hand, the molecular finding of SLC34A1 mutations in the clinical setting of the FRTS2 was achieved during the reassessment of two patients previously evaluated at the clinical level by Tieder et al. [79]: 12 years later, these patients were found to be homozygote carriers of the SLC34A1 variant c.460_480dup (p.Ile154_Val160dup) [78]. Furthermore, many case reports described SLC34A1-mutated subjects in the clinical context of hypophosphatemia with Pi wasting and kidney stones. Compound heterozygosis in SLC34A1 was also described in an infant with $\mathrm{NC}$ and primary hyperoxaluria carrying the two missense variants c.271_291del

Table 3: Loss-of-function variants of SLC34A1 associated to non-IIH phenotype.

\begin{tabular}{|c|c|c|c|c|c|}
\hline Exon/intron & $\begin{array}{l}\text { Genomic position } \\
\text { (NG_016223.1) }\end{array}$ & $\begin{array}{l}\text { Transcript position } \\
\text { (NM_003052.4) }\end{array}$ & $\begin{array}{l}\text { Protein change } \\
\text { (NP_003043.3) }\end{array}$ & $\begin{array}{l}\text { Clinical } \\
\text { phenotype }\end{array}$ & Ref \\
\hline 3 & g.6589_6590delinsTT & c.142_143delinsTT & p.(Ala48Phe) & NPHLOP1 & [75] \\
\hline 5 & g.7002C $>\mathrm{T}$ & c. $398 \mathrm{C}>\mathrm{T}$ & p.(Ala133Val) & $\mathrm{HCu}, \mathrm{hPi}, \mathrm{NL}$ & [85] \\
\hline 5 & g.7041C>T & $c .437 \mathrm{C}>\mathrm{T}$ & p.(Pro146Leu) & $\mathrm{HPi}, \mathrm{NL}$ & [85] \\
\hline 5 & g.7043G >A & c. $439 \mathrm{G}>\mathrm{A}$ & p.(Val147Met) & NPHLOP1 & [75] \\
\hline 5 & g.7064_7084dup & c.460_480dup & p.(Ile154_Val160dup) & FRTS2 & [78] \\
\hline 6 & g. $8335 \mathrm{~T}>\mathrm{C}$ & c. $536 \mathrm{~T}>\mathrm{C}$ & p.(Leu179Pro) & NC & [92] \\
\hline 12 & g.17576G $>A$ & c. $1348 \mathrm{G}>\mathrm{A}$ & p.(Gly450Ser) & $\mathrm{HPi}, \mathrm{HCu}, \mathrm{NL}$ & [85] \\
\hline 12 & g.17595T $>A$ & c.1367T $>A$ & p.(Ile456Asn) & $\mathrm{HCu}, \mathrm{NL}$ & [85] \\
\hline 13 & g. $18402 A>G$ & c. $1466 \mathrm{~A}>\mathrm{G}$ & p.(Tyr489Cys) & NL, low PTH, hPi & [118] \\
\hline 13 & g.18470C $>\mathrm{T}$ & c. $1534 C>T$ & p.(Arg512Cys) & NC & [116] \\
\hline 13 & g.18660C $>\mathrm{T}$ & c. $1724 C>T$ & p.(Thr575lle) & $\mathrm{NL}, \mathrm{Hca}$ & [92] \\
\hline
\end{tabular}

All the mutations in SLC34A1 associated with other then IIH phenotype are reported. NPHLOP1, hypophosphatemic nephrolithiasis with osteoporosis, type 1; FRTS2, Fanconi renotubular syndrome, type 2; HCa, hypercalcemia; HCu, hypercalciuria; hPi, hypophosphatemia; NL, nephrolithiasis; NC, nephrocalcinosis; PTH, parathyroid hormone. 
(p.Val91_Ala97del) and c.1534C>T (p.Arg512Cys) [116]. SLC34A1 was also associated with recurrent kidney stones disease in a large Icelandic genome analysis consortium where the missense variant c.1466A $>\mathrm{G}$ (p.Tyr489Cys) was associated with hypophosphatemia, NL and low PTH level [118]. Successively, Braun et al. described, in a cohort of 143 pediatric individuals, several heterozygote variants in the SLC34A1 gene associated with clinical features of NL, hypercalciuria and hypophosphatemia. Despite their apparent dominant effect, this paper did not provide any definitive explanation about the pathogenic role of each allele variant [85]. In this regard, Dinour et al. described clinical evidence of $\mathrm{NC}$ and kidney failure only in the homozygous carriers belonging to different members of Israeli-Arab families [90]. Moreover, Daga et al. recently evaluated a cohort of early-onset NL/NC and described both mono- and bi-allelic variants in the SLC34A1 gene: the author reported two cases of recessive transmission associated with the compound heterozygosis of the $\mathrm{c} \cdot 644+1 \mathrm{G}>\mathrm{A}$ splice site variant with the c.1204G $>$ C (p.Gly402Arg) mutation or the homozygote c.1724C $>\mathrm{T}$ (p.Thr575Ile) missense change, respectively. Other two missense variants were described in heterozygosis, the c.398C $>\mathrm{T}$ (p.Ala133Val) and the c.536T $>$ C (p.Leu179Pro). Notably, the individuals harboring heterozygotes variants exhibited NC, hypercalcemia, hypercalciuria and hypophosphatemia consistent with renal Pi wasting. On the other hand, affected individuals carrying the dominant mutations showed $\mathrm{NC}$ and hypercalciuria without hypercalcemia. The authors highlighted that a dichotomy based on the age of onset could characterize SLC34A1 genetic variants: recessive cases are typically reported in infancy (median age: 0.25 years) whereas dominant cases tend to present later in childhood (median age: 3.5 years) [92]. Recently, Fearn et al. reported two patients with metabolic acidosis, hyperphosphaturia and renal stones: this clinical phenotype occurring in both dominant and recessive patterns. Overall, an unclear pathogenic role of mono- and bi-allelic loss of function mutations in SLC34A1 genes has emerged [74].

\section{Clinical and diagnostic relevance}

IIH is an uncommon disorder and a high degree of misdiagnosis can occur. The genotyping of CYP24A1 and SLC34A1 genes is considered the definitive step in the diagnosis of this condition and, consequently, all patients with a suspected IIH phenotype should be addressed to the molecular testing. A genetic basis of IIH should be particularly taken into account in the case of early-onset, familial prevalence, familial consanguinity and a history of multiple or recurrent kidney stones [85]. Across the availability of molecular high-throughput approaches, the mutation assay could be evaluated not only as the final step into a diagnostic workflow but also as the viable and cost-effective screening tool $[116,119]$. At present, genetic testing is recommended but not widely accessible to all patients and clinicians, due to both the lack of available genetic counseling and to the need of specialized laboratories. The efficient identification of both infantile and adult forms of CYP24A1 or SLC34A1 deficiency has a great impact on the patient's management in terms of appropriate treatment and diet modification. In IIH patients, the source of vitamin D, such as supplementation therapy, is removed and a low-Ca diet is introduced. In the case of defective NaPi-IIa, Pi supplementation is the targeted therapeutic approach, aimed at restoring the serum Pi levels and at normalizing vitamin $\mathrm{D}$ and Ca metabolism [3, 66, 91]. In this sense, early and correct molecular diagnosis may be crucial for the setting of the optimal feedback therapy in response to the primary genetic defect [81]. The pharmacological treatments that can be administrated in IIH patients are mainly focused on the avoidance of long-lasting and recurrent hypercalcemic episodes [120]. Several therapeutic approaches can be applied, principally focused on the decrease of intestinal Ca absorption, the promotion of renal Ca excretion, the reduction of Ca release from bone and the inhibition of the CYP27B1 activity [121]. Common treatments include the administration of steroids, bisphosphonates, calcitonin and glucocorticoids that can be given if the symptomatic hypercalcemia persists despite hydration and diuretics [21]. Bisphosphonates inhibit osteoclast activity and osteocytes apoptosis, indirectly stimulating the osteoblast function [122]. Administration of this drug has been described as a successful treatment in an infant with severe hypercalcemia due to a CYP24A1 genetic variant, however, a long-term evaluation and more extensive clinical trials are necessary to understand the beneficial and side effects of this therapy in CYP24A1 mutation carriers [123]. On the contrary, long-term steroids administration seems not to be an efficient therapy for IIH, as in some patients with CYP24A1 genetic variants the hypercalcemia persists after pharmacological treatment [124]. A successful use of the azole agents is reported as a specific targeted pharmacological therapy of IIH caused by CYP24A1 loss of function. This class of drugs, primarily antifungal agents, is specifically used in vitamin D-mediated hypercalcemia as general inhibitors of cytochrome P450-enzymes. These drugs are heterocyclic ring compounds classified as imidazoles (e.g. ketoconazole) or triazoles (e.g. fluconazole), containing two or three nitrogen atoms in the azole ring, 
respectively. In particular, ketoconazole was successfully used in patients with CYP24A1 impairment showing an effective normalization of biochemical parameters [53, 58, 125, 126]. However, long-term and high-dose treatment ketoconazole may be associated with several adverse effects such as renal and hepatic toxicity and a protracted administration is not always advisable. Fluconazole, however, shows a lower inhibitory activity, is well tolerated due to reduced side effects and, consequently, it should be considered as an alternative treatment [127]. Another therapeutic approach proposed in IIH treatment foresees the induction of the CYP3A4 activity through administration of rifampin. CYP3A4 is a $\mathrm{P} 450$ enzyme that inactivates many drugs and may represent an alternative pathway for the inactivation of the active vitamin D metabolites [128]. Notably, the long-term monitoring through dietary modification and biochemical follow-up represents a crucial issue for avoiding relapses and acute exacerbation of the disease. Generally, while hypercalcemia resolves during follow-up, persistent hypercalciuria is a common finding [129]: as a consequence, the main goal of long-term evaluation is the prevention of renal deterioration and its associated complications.

\section{Conclusions and future prospects}

The recent association between CYP24A1 and SLC34A1 variants with IIH syndrome have piqued the interest of laboratory professionals, geneticists and clinicians, paving the way for a progressive growth of literature case reports. If acquired conditions are the main causes of hypercalcemia in adults, inherited disorders and vitamin D intoxications are the most prevalent in infancy and require more attention in the diagnostic evaluation. Notably, in IIH patients the clinical outcome could also be triggered by the administration of vitamin $\mathrm{D}$. The public belief about the health benefits of high levels of vitamin $\mathrm{D}$, together with the prophylactic measures aimed at the prevention of rickets, has led to a common use of supplementation schemes. IIH infants receiving high doses of vitamin D are more vulnerable to toxicity: therefore, a tailored dosage regimen is required in the view of personalized medicine. In this scenario, the relevance of genetic testing is emerging dramatically. In early-onset $\mathrm{NC} / \mathrm{NL}$ subjects and before prescribing vitamin D supplementation therapy, molecular analysis should be routinely taken into account in both adults and infants to rule out abnormalities in $\mathrm{Ca}$ and Pi metabolism. The molecular evaluation allows the definitive diagnosis of IIH also suggesting the specific
IIH subtype. This has lead to a significant improvement of medical evaluations, with practical consequences in patients' care in terms of tailored treatments. The introduction of the genetic testing for CYP24A1 and SLC34A1 offers a valuable tool to fully understand the vitamin D and Pi metabolic pathways of each patient. Moreover, clinicians would mainly benefit from these results in the forthcoming monitoring of infants as well as adult patients in the context of personalized and precision medicine. Finally, the possibility to reduce the risk of future chronic manifestations represents an important aspect of lifestyle quality of patients and could reflect on a wider scale the efficiency of public health.

Author contributions: All the authors have accepted responsibility for the entire content of this submitted manuscript and approved submission.

Research funding: None declared.

Employment or leadership: None declared.

Honorarium: None declared.

Competing interests: The funding organization(s) played no role in the study design; in the collection, analysis, and interpretation of data; in the writing of the report; or in the decision to submit the report for publication.

Conflict of interest: None declared.

Ethical approval: The conducted research is not related to either human or animal use.

\section{References}

1. Marcinowska-Suchowierska E, Kupisz-Urbańska M, Łukaszkiewicz J, Płudowski P, Jones G. Vitamin D toxicity-a clinical perspective. Front Endocrinol (Lausanne) 2018;9:550.

2. Schlingmann KP, Kaufmann M, Weber S, Irwin A, Goos C, John U, et al. Mutations in CYP24A1 and idiopathic infantile hypercalcemia. N Engl J Med 2011;365:410-21.

3. Schlingmann KP, Ruminska J, Kaufmann M, Dursun I, Patti M, Kranz B, et al. Autosomal-recessive mutations in SLC34A1 encoding sodium-phosphate cotransporter $2 \mathrm{~A}$ cause idiopathic infantile hypercalcemia. J Am Soc Nephrol 2016;27:604-14.

4. Mohebbi N, Ferraro PM, Gambaro G, Unwin R. Tubular and genetic disorders associated with kidney stones. Urolithiasis 2017;45:127-37.

5. Masuda S, Byford V, Arabian A, Sakai Y, Demay MB, St-Arnaud R, et al. Altered pharmacokinetics of 1alpha,25-dihydroxyvitamin D3 and 25-hydroxyvitamin D3 in the blood and tissues of the 25-hydroxyvitamin D-24-hydroxylase (Cyp24a1) null mouse. Endocrinology 2005;146:825-34.

6. Veldurthy V, Wei R, Campbell M, Lupicki K, Dhawan P, Christakos S. 25-Hydroxyvitamin $\mathrm{D}$ (3) 24-hydroxylase: a key regulator of $1,25(\mathrm{OH})(2) \mathrm{D}(3)$ catabolism and calcium homeostasis. Vitam Horm 2016;100:137-50. 
7. Jones G, Prosser DE, Kaufmann M. 25-Hydroxyvitamin D-24-hydroxylase (CYP24A1): its important role in the degradation of vitamin D. Arch Biochem Biophys 2012;523:9-18.

8. Prosser DE, Jones G. Enzymes involved in the activation and inactivation of vitamin D. Trends Biochem Sci 2004;29:664-73.

9. Jones G, Prosser DE, Kaufmann M. Cytochrome P450-mediated metabolism of vitamin D. J Lipid Res 2014;55:13-31.

10. Kaufmann M, Lee SM, Pike JW, Jones G. A high-calcium and phosphate rescue diet and VDR-expressing transgenes normalize serum vitamin D metabolite profiles and renal Cyp27b1 and Cyp24a1 expression in VDR null mice. Endocrinology 2015;156:4388-97.

11. Tanaka Y, DeLuca HF. Measurement of mammalian 25-hydroxyvitamin D3 24R-and 1 alpha-hydroxylase. Proc Natl Acad Sci U S A 1981;78:196-9.

12. Shinki T, Jin CH, Nishimura A, Nagai Y, Ohyama Y, Noshiro $M$, et al. Parathyroid hormone inhibits 25 -hydroxyvitamin D3-24-hydroxylase mRNA expression stimulated by 1 alpha,25dihydroxyvitamin D3 in rat kidney but not in intestine. J Biol Chem 1992;267:13757-62.

13. Shigematsu T, Horiuchi N, Ogura Y, Miyahara T, Suda T. Human parathyroid hormone inhibits renal 24-hydroxylase activity of 25-hydroxyvitamin D3 by a mechanism involving adenosine 3',5'-monophosphate in rats. Endocrinology 1986;118:1583-9.

14. Armbrecht HJ, Hodam TL, Boltz MA. Hormonal regulation of 25-hydroxyvitamin D3-1alpha-hydroxylase and 24-hydroxylase gene transcription in opossum kidney cells. Arch Biochem Biophys 2003;409:298-304.

15. Zierold C, Reinholz GG, Mings JA, Prahl JM, DeLuca HF. Regulation of the procine 1,25-dihydroxyvitamin D3-24-hydroxylase (CYP24) by 1,25-dihydroxyvitamin D3 and parathyroid hormone in AOK-B50 cells. Arch Biochem Biophys 2000;381:323-7.

16. Zierold C, Mings JA, DeLuca HF. Parathyroid hormone regulates 25-hydroxyvitamin $D(3)$-24-hydroxylase mRNA by altering its stability. Proc Natl Acad Sci U S A 2001;98:13572-6.

17. Zierold C, Mings JA, DeLuca HF. Regulation of 25-hydroxyvitamin D3-24-hydroxylase mRNA by 1,25-dihydroxyvitamin D3 and parathyroid hormone. J Cell Biochem 2003;88:234-7.

18. Wu W, Fan H, Jiang Y, Liao L, Li L, Zhao J, et al. Regulation of 25-hydroxyvitamin D-1-hydroxylase and 24-hydroxylase in keratinocytes by PTH and FGF23. Exp Dermatol 2018;27: 1201-9.

19. St-Arnaud R, Arabian A, Travers R, Barletta F, Raval-Pandya $M$, Chapin K, et al. Deficient mineralization of intramembranous bone in vitamin D-24-hydroxylase-ablated mice is due to elevated 1,25-dihydroxyvitamin D and not to the absence of 24,25-dihydroxyvitamin D. Endocrinology 2000;141:2658-66.

20. Christakos S, Dhawan P, Verstuyf A, Verlinden L, Carmeliet G. Vitamin D: metabolism, molecular mechanism of action, and pleiotropic effects. Physiol Rev 2016;96:365-408.

21. Vogiatzi MG, Jacobson-Dickman E, DeBoer MD, Drugs and Therapeutics Committee of The Pediatric Endocrine Society. Vitamin D supplementation and risk of toxicity in pediatrics: a review of current literature. J Clin Endocrinol Metab 2104;99:1132-41.

22. Wagner CA, Hernando N, Forster IC, Biber J. The SLC34 family of sodium-dependent phosphate transporters. Pflugers Arch 2014;466:139-53.

23. Kido S, Kaneko I, Tatsumi S, Segawa H, Miyamoto K. Vitamin D and type II sodium-dependent phosphate cotransporters. Contrib Nephrol 2013;180:86-97.
24. Berndt T, Kumar R. Phosphatonins and the regulation of phosphate homeostasis. Annu Rev Physiol 2007;69:341-59.

25. Custer M, Lotscher M, Biber J, Murer H, Kaissling B. Expression of Na-P(i) cotransport in rat kidney: localization by RT-PCR and immunohistochemistry. Am J Physiol 1994;266(5 Pt 2):F767-74.

26. Murer H. Functional domains in the renal type Ila Na/P(i)cotransporter. Kidney Int 2002;62:375-82.

27. Antoniucci DM, Yamashita T, Portale AA. Dietary phosphorus regulates serum fibroblast growth factor-23 concentrations in healthy men. J Clin Endocrinol Metab 2006;91:3144-9.

28. Murer H, Hernando N, Forster I, Biber J. Proximal tubular phosphate reabsorption: molecular mechanisms. Physiol Rev 2000;80:1373-409.

29. Navarro-Garcia JA, Fernandez-Velasco M, Delgado C, Delgado JF, Kuro OM, Ruilope LM, et al. PTH, vitamin D, and the FGF-23klotho axis and heart: going beyond the confines of nephrology. Eur J Clin Invest 2018;48:e12902.

30. Taketani Y, Segawa H, Chikamori M, Morita K, Tanaka K, Kido S, et al. Regulation of type II renal $\mathrm{Na}^{+}$-dependent inorganic phosphate transporters by 1,25-dihydroxyvitamin D3. Identification of a vitamin D-responsive element in the human NAPi-3 gene. J Biol Chem 1998;273:14575-81.

31. Marks J, Srai SK, Biber J, Murer H, Unwin RJ, Debnam ES. Intestinal phosphate absorption and the effect of vitamin D: a comparison of rats with mice. Exp Physiol 2006;91:531-7.

32. Friedlaender MM, Wald H, Dranitzki-Elhalel M, Zajicek HK, Levi $\mathrm{M}$, Popovtzer MM. Vitamin D reduces renal NaPi-2 in PTH-infused rats: complexity of vitamin $\mathrm{D}$ action on renal $\mathrm{P}(\mathrm{i})$ handling. Am J Physiol Renal Physiol 2001;281:F428-33.

33. Kaneko I, Segawa H, Furutani J, Kuwahara S, Aranami F, Hanabusa $E$, et al. Hypophosphatemia in vitamin $D$ receptor null mice: effect of rescue diet on the developmental changes in renal $\mathrm{Na}^{+}$-dependent phosphate cotransporters. Pflugers Arch 2011;461:77-90.

34. Huang X, Jiang Y, Xia W. FGF23 and phosphate wasting disorders. Bone Res 2103;1:120-32.

35. Forster IC, Hernando N, Biber J, Murer H. Proximal tubular handling of phosphate: a molecular perspective. Kidney Int 2006;70:1548-59.

36. Perwad F, Azam N, Zhang MY, Yamashita T, Tenenhouse HS, Portale AA. Dietary and serum phosphorus regulate fibroblast growth factor 23 expression and 1,25-dihydroxyvitamin D metabolism in mice. Endocrinology 2005;146:5358-64.

37. Perwad F, Portale AA. Vitamin D metabolism in the kidney: regulation by phosphorus and fibroblast growth factor 23. Mol Cell Endocrinol 2011;347:17-24.

38. Lightwood R, Stapleton T. Idiopathic hypercalcaemia in infants. Lancet 1953;265:255-6.

39. Pronicka E, Kulczycka H, Rowinska E, Konopinska A, Kansy J, Lorenc R. Idiopathic hypercalcemia as a syndrome of hypersensitivity to vitamin D3 in 19 infants. Pediatr Pol 1985;60: 288-94.

40. Misselwitz J, Hesse V, Markestad T. Nephrocalcinosis, hypercalciuria and elevated serum levels of 1,25-dihydroxyvitamin D in children. Possible link to vitamin D toxicity. Acta Paediatr Scand 1990;79:637-43.

41. Hooft C, Vermassen A, Eeckels R, Vanheule R. Familial incidence of hypercalcaemia. Extreme hypersensitivity to vitamin $D$ in an infant whose father suffered from sarcoidosis. Helv Paediatr Acta 1961;16:199-210. 
42. Mehes K, Szelid Z, Toth P. Possible dominant inheritance of the idiopathic hypercalcemic syndrome. Hum Hered 1975;25:30-4.

43. Smith DW, Blizzard RM, Harrison HE. Idiopathic hypercalcemia; a case report with assays of vitamin $D$ in the serum. Pediatrics 1959;24:258-69.

44. Madsen JO, Sauer S, Beck B, Johannesen J. CYP24A1 mutation in a girl infant with idiopathic infantile hypercalcemia. J Clin Res Pediatr Endocrinol 2018;10:83-6.

45. Fencl F, Blahova K, Schlingmann KP, Konrad M, Seeman T. Severe hypercalcemic crisis in an infant with idiopathic infantile hypercalcemia caused by mutation in CYP24A1 gene. Eur J Pediatr 2103;172:45-9.

46. Gut J, Kutilek S. Idiopathic infantile hypercalcaemia in 5-month old girl. Prague Med Rep 2011;112:124-31.

47. Dowen FE, Sayers JA, Hynes AM, Sayer JA. CYP24A1 mutation leading to nephrocalcinosis. Kidney Int 2104;85:1475.

48. Jobst-Schwan T, Pannes A, Schlingmann KP, Eckardt KU, Beck $B B$, Wiesener MS. Discordant clinical course of vitamin-Dhydroxylase (CYP24A1) associated hypercalcemia in two adult brothers with nephrocalcinosis. Kidney Blood Press Res 2105; 40:443-51.

49. Wolf $P$, Muller-Sacherer T, Baumgartner-Parzer S, Winhofer $\mathrm{Y}$, Kroo J, Gessl A, et al. A case of "Late-Onset" idiopathic infantile hypercalcemia secondary to mutations in the CYP24A1 gene. Endocr Pract 2104;20:e91-5.

50. Baudart P, Molin A, Cesini J, Jones G, Kaufmann M, Kottler ML, et al. Calcium pyrophosphate deposition disease revealing a hypersensitivity to vitamin D. Joint Bone Spine 2107;84:349-51.

51. Ferraro PM, Minucci A, Primiano A, De Paolis E, Gervasoni J, Persichilli S, et al. A novel CYP24A1 genotype associated to a clinical picture of hypercalcemia, nephrolithiasis and low bone mass. Urolithiasis 2107;45:291-4.

52. Figueres ML, Linglart A, Bienaime F, Allain-Launay E, RousseyKessler G, Ryckewaert A, et al. Kidney function and influence of sunlight exposure in patients with impaired 24-hydroxylation of vitamin D due to CYP24A1 mutations. Am J Kidney Dis 2105;65:122-6.

53. Nesterova G, Malicdan MC, Yasuda K, Sakaki T, Vilboux T, Ciccone C, et al. 1,25-(OH)2D-24 hydroxylase (CYP24A1) deficiency as a cause of nephrolithiasis. Clin J Am Soc Nephrol 2103;8:649-57.

54. Jing J, Pattaro C, Hoppmann A, Okada Y, Consortium CK, Fox CS, et al. Combination of mouse models and genomewide association studies highlights novel genes associated with human kidney function. Kidney Int 2016;90:764-73.

55. Carpenter TO. CYP24A1 loss of function: clinical phenotype of monoallelic and biallelic mutations. J Steroid Biochem Mol Biol 2017;173:337-40.

56. Cools M, Goemaere S, Baetens D, Raes A, Desloovere A, Kaufman JM, et al. Calcium and bone homeostasis in heterozygous carriers of CYP24A1 mutations: a cross-sectional study. Bone 2015;81:89-96.

57. Colussi G, Ganon L, Penco S, De Ferrari ME, Ravera F, Querques $M$, et al. Chronic hypercalcaemia from inactivating mutations of vitamin D 24-hydroxylase (CYP24A1): implications for mineral metabolism changes in chronic renal failure. Nephrol Dial Transplant 2014;29:636-43.

58. Tebben PJ, Milliner DS, Horst RL, Harris PC, Singh RJ, Wu Y, et al. Hypercalcemia, hypercalciuria, and elevated calcitriol concentrations with autosomal dominant transmission due to
CYP24A1 mutations: effects of ketoconazole therapy. J Clin Endocrinol Metab 2012;97:E423-7.

59. Molin A, Baudoin R, Kaufmann M, Souberbielle JC, Ryckewaert A, Vantyghem MC, et al. CYP24A1 mutations in a cohort of hypercalcemic patients: evidence for a recessive trait. J Clin Endocrinol Metab 2015;100:E1343-52.

60. Dinour D, Beckerman P, Ganon L, Tordjman K, Eisenstein Z, Holtzman EJ. Loss-of-function mutations of CYP24A1, the vitamin D 24-hydroxylase gene, cause long-standing hypercalciuric nephrolithiasis and nephrocalcinosis. J Urol 2013;190:552-7.

61. O'Keeffe DT, Tebben PJ, Kumar R, Singh RJ, Wu Y, Wermers RA. Clinical and biochemical phenotypes of adults with monoallelic and biallelic CYP24A1 mutations: evidence of gene dose effect. Osteoporos Int 2016;27:3121-5.

62. Woods GN, Saitman A, Gao H, Clarke NJ, Fitzgerald RL, Chi NW. A young woman with recurrent gestational hypercalcemia and acute pancreatitis caused by CYP24A1 deficiency. J Bone Miner Res 2016;31:1841-4.

63. Karras SN, Wagner CL, Castracane VD. Understanding vitamin D metabolism in pregnancy: From physiology to pathophysiology and clinical outcomes. Metabolism 2018;86:112-23.

64. Novakovic B, Sibson M, Ng HK, Manuelpillai U, Rakyan V, Down T, et al. Placenta-specific methylation of the vitamin D 24-hydroxylase gene: implications for feedback autoregulation of active vitamin D levels at the fetomaternal interface. J Biol Chem 2009;284:14838-48.

65. Dinour D, Davidovits M, Aviner S, Ganon L, Michael L, ModanMoses D, et al. Maternal and infantile hypercalcemia caused by vitamin-D-hydroxylase mutations and vitamin D intake. Pediatr Nephrol 2015;30:145-52.

66. Wagner CA, Rubio-Aliaga I, Hernando N. Renal phosphate handling and inherited disorders of phosphate reabsorption: an update. Pediatr Nephrol 2019;34:549-59.

67. Arcidiacono T, Mingione A, Macrina L, Pivari F, Soldati L, Vezzoli G. Idiopathic calcium nephrolithiasis: a review of pathogenic mechanisms in the light of genetic studies. Am J Nephrol 2014;40:499-506.

68. Urabe Y, Tanikawa C, Takahashi A, Okada Y, Morizono T, Tsunoda T, et al. A genome-wide association study of nephrolithiasis in the Japanese population identifies novel susceptible Loci at 5q35.3, 7p14.3, and 13q14.1. PLoS Genet 2012;8:e1002541.

69. Beck L, Karaplis AC, Amizuka N, Hewson AS, Ozawa H, Tenenhouse HS. Targeted inactivation of Npt2 in mice leads to severe renal phosphate wasting, hypercalciuria, and skeletal abnormalities. Proc Natl Acad Sci U S A 1998;95:5372-7.

70. Tenenhouse HS. Regulation of phosphorus homeostasis by the type iia na/phosphate cotransporter. Annu Rev Nutr 2005;25:197-214.

71. Chau H, El-Maadawy S, McKee MD, Tenenhouse HS. Renal calcification in mice homozygous for the disrupted type Ila $\mathrm{Na} / \mathrm{Pi}$ cotransporter gene Npt2. J Bone Miner Res 2003;18:644-57.

72. Khan SR, Canales BK. Ultrastructural investigation of crystal deposits in Npt2a knockout mice: are they similar to human Randall's plaques? J Urol 2011;186:1107-13.

73. Iwaki T, Sandoval-Cooper MJ, Tenenhouse HS, Castellino FJ. A missense mutation in the sodium phosphate co-transporter Slc34a1 impairs phosphate homeostasis. J Am Soc Nephrol 2008;19:1753-62. 
74. Fearn A, Allison B, Rice SJ, Edwards N, Halbritter J, Bourgeois S, et al. Clinical, biochemical, and pathophysiological analysis of SLC34A1 mutations. Physiol Rep 2018;6:e13715.

75. Priè D, Huart V, Bakouh N, Planelles G, Dellis 0 , Gerard B, et al. Nephrolithiasis and osteoporosis associated with hypophosphatemia caused by mutations in the type 2a sodium-phosphate cotransporter. N Engl J Med 2002;347:983-91.

76. Lapointe JY, Tessier J, Paquette Y, Wallendorff B, Coady MJ, Pichette V, et al. NPT2a gene variation in calcium nephrolithiasis with renal phosphate leak. Kidney Int 2006;69: 2261-7.

77. Virkki LV, Forster IC, Hernando N, Biber J, Murer H. Functional characterization of two naturally occurring mutations in the human sodium-phosphate cotransporter type Ila. J Bone Miner Res 2003;18:2135-41.

78. Magen D, Zelikovic I, Skorecki K. Genetic disorders of renal phosphate transport. N Engl I Med 2010;363:1774; author reply 1774-5.

79. Tieder M, Arie R, Modai D, Samuel R, Weissgarten J, Liberman UA. Elevated serum 1,25-dihydroxyvitamin D concentrations in siblings with primary Fanconi's syndrome. N Engl J Med 1988;319:845-9.

80. Kenny J, Lees MM, Drury S, Barnicoat A, Van't Hoff W, Palmer $R$, et al. Sotos syndrome, infantile hypercalcemia, and nephrocalcinosis: a contiguous gene syndrome. Pediatr Nephrol 2011;26:1331-4.

81. Pronicka E, Ciara E, Halat P, Janiec A, Wojcik M, Rowinska E, et al. Biallelic mutations in CYP24A1 or SLC34A1 as a cause of infantile idiopathic hypercalcemia (IIH) with vitamin $D$ hypersensitivity: molecular study of 11 historical IIH cases. J Appl Genet 2017;58:349-53.

82. Gigante M, Santangelo L, Diella S, Caridi G, Argentiero L, D'Alessandro MM, et al. Mutational spectrum of CYP24A1 gene in a cohort of Italian patients with idiopathic infantile hypercalcemia. Nephron 2016;133:193-204.

83. Meusburger E, Mundlein A, Zitt E, Obermayer-Pietsch B, Kotzot $D$, Lhotta K. Medullary nephrocalcinosis in an adult patient with idiopathic infantile hypercalcaemia and a novel CYP24A1 mutation. Clin Kidney J 2013;6:211-5.

84. Tang JC, Nicholls H, Piec I, Washbourne CJ, Dutton JJ, Jackson $\mathrm{S}$, et al. Reference intervals for serum 24,25-dihydroxyvitamin $D$ and the ratio with 25 -hydroxyvitamin D established using a newly developed LC-MS/MS method. J Nutr Biochem 2017;46:21-9.

85. Braun DA, Lawson JA, Gee HY, Halbritter J, Shril S, Tan W, et al. Prevalence of monogenic causes in pediatric patients with nephrolithiasis or nephrocalcinosis. Clin J Am Soc Nephrol 2016;11:664-72.

86. Castanet M, Mallet E, Kottler ML. Lightwood syndrome revisited with a novel mutation in CYP24 and vitamin D supplement recommendations. J Pediatr 2013;163:1208-10.

87. Molin A, Nowoczyn M, Coudray N, Ballandone C, Abéguilé G, Mittre $\mathrm{H}$, et al. Molecular characterization of a recurrent $10.9 \mathrm{~kb}$ CYP24A1 deletion in Idiopathic Infantile Hypercalcemia. Eur J Med Genet 2018; doi: 10.1016/j.ejmg.2018.11.011.

88. Ashton EJ, Legrand A, Benoit V, Roncelin I, Venisse A, Zennaro MC, et al. Simultaneous sequencing of 37 genes identified causative mutations in the majority of children with renal tubulopathies. Kidney Int 2018;93:961-7.
89. Demir K, Yildiz M, Bahat H, Goldman M, Hassan N, Tzur S, et al. Clinical heterogeneity and phenotypic expansion of NaPi-Ilaassociated disease. J Clin Endocrinol Metab 2017;102:4604-14.

90. Dinour D, Davidovits M, Ganon L, Ruminska J, Forster IC, Hernando N, et al. Loss of function of NaPilla causes nephrocalcinosis and possibly kidney insufficiency. Pediatr Nephrol 2016;31:2289-97.

91. Kurnaz E, Erdeve Ş, Çetinkaya S, Aycan Z. Rare cause of infantile hypercalcemia: a novel mutation in the SLC34A1 gene. Horm Res Paediatr 2018;18:1-7.

92. Daga A, Majmundar AJ, Braun DA, Gee HY, Lawson JA, Shril S, et al. Whole exome sequencing frequently detects a monogenic cause in early onset nephrolithiasis and nephrocalcinosis. Kidney Int 2018;93:204-13.

93. Hureaux M, Molin A, Jay N, Saliou AH, Spaggiari E, Salomon R, et al. Prenatal hyperechogenic kidneys in three cases of infantile hypercalcemia associated with SLC34A1 mutations. Pediatr Nephrol 2018;33:1723-9.

94. Rajagopal A, Braslavsky D, Lu JT, Kleppe S, Clement F, Cassinelli $\mathrm{H}$, et al. Exome sequencing identifies a novel homozygous mutation in the phosphate transporter SLC34A1 in hypophosphatemia and nephrocalcinosis. J Clin Endocrinol Metab 2014;99:E2451-6.

95. Fokkema IF, Taschner PE, Schaafsma GC, Celli J, Laros JF, den Dunnen JT. LOVD v.2.0: the next generation in gene variant databases. Hum Mutat 2011;32:557-63.

96. Stenson PD, Ball EV, Mort M, Phillips AD, Shiel JA, Thomas NS, et al. Human gene mutation database (HGMD): 2003 update. Hum Mutat 2003;21:577-81.

97. Landrum MJ, Lee JM, Benson M, Brown GR, Chao C, Chitipiralla $\mathrm{S}$, et al. ClinVar: improving access to variant interpretations and supporting evidence. Nucleic Acids Res 2018;46(D1):D1062-7.

98. den Dunnen JT, Dalgleish R, Maglott DR, Hart RK, Greenblatt MS, McGowan-Jordan J, et al. HGVS recommendations for the description of sequence variants: 2016 update. Hum Mutat 2016;37:564-9.

99. Wildeman M, van Ophuizen E, den Dunnen JT, Taschner PE. Improving sequence variant descriptions in mutation databases and literature using the Mutalyzer sequence variation nomenclature checker. Hum Mutat 2008;29:6-13.

100. Annalora AJ, Goodin DB, Hong WX, Zhang Q, Johnson EF, Stout CD. Crystal structure of CYP24A1, a mitochondrial cytochrome P450 involved in vitamin D metabolism. J Mol Biol 2010;396:441-51.

101. Fenollar-Ferrer C, Patti M, Knopfel T, Werner A, Forster IC, Forrest LR. Structural fold and binding sites of the human $\mathrm{Na}(+)$-phosphate cotransporter $\mathrm{NaPi}$-II. Biophys J 2014;106:1268-79.

102. Sherry ST, Ward MH, Kholodov M, Baker J, Phan L, Smigielski EM. dbSNP: the NCBI database of genetic variation. Nucleic Acids Res 2001;29:308-11.

103. Adzhubei IA, Schmidt S, Peshkin L, Ramensky VE, Gerasimova A, Bork P, et al. A method and server for predicting damaging missense mutations. Nat Methods 2010;7:248-9.

104. Schwarz JM, Cooper DN, Schuelke M, Seelow D. MutationTaster2: mutation prediction for the deep-sequencing age. Nat Methods 2014;11:361-2.

105. Kumar P, Henikoff S, Ng PC. Predicting the effects of coding non-synonymous variants on protein function using the SIFT algorithm. Nat Protoc 2009;4:1073-81. 
106. Desmet FO, Hamroun D, Lalande M, Collod-Beroud G, Claustres $M$, Beroud C. Human Splicing Finder: an online bioinformatics tool to predict splicing signals. Nucleic Acids Res 2009;37:e67.

107. Zhou X, Edmonson MN, Wilkinson MR, Patel A, Wu G, Liu Y, et al. Exploring genomic alteration in pediatric cancer using ProteinPaint. Nat Genet 2016;48:4-6.

108. Moreland JL, Gramada A, Buzko OV, Zhang Q, Bourne PE. The molecular biology toolkit (MBT): a modular platform for developing molecular visualization applications. BMC Bioinformatics 2005;6:21.

109. Nagamani S, Singh Kh D, Muthusamy K. Combined sequence and sequence-structure based methods for analyzing FGF23, CYP24A1 and VDR genes. Meta Gene 2016;9:26-36.

110. Ji HF, Shen L. CYP24A1 mutations in idiopathic infantile hypercalcemia. N Engl J Med 2011;365:1741; author reply 1742-3.

111. Mugg A, Legeza B, Tee MK, Damm I, Long RK, Miller WL. Quantitation of CYP24A1 enzymatic activity with a simple two-hybrid system. J Clin Endocrinol Metab 2015;100:684-8.

112. Streeten EA, Zarbalian K, Damcott CM. CYP24A1 mutations in idiopathic infantile hypercalcemia. N Engl J Med 2011;365:1741-2; author reply 1742-3.

113. Masuda S, Prosser DE, Guo YD, Kaufmann M, Jones G. Generation of a homology model for the human cytochrome P450, CYP24A1, and the testing of putative substrate binding residues by site-directed mutagenesis and enzyme activity studies. Arch Biochem Biophys 2007;460:177-91.

114. Dauber A, Nguyen TT, Sochett E, Cole DE, Horst R, Abrams SA, et al. Genetic defect in CYP24A1, the vitamin D 24-hydroxylase gene, in a patient with severe infantile hypercalcemia. J Clin Endocrinol Metab 2012;97:E268-74.

115. Annalora AJ, Bobrovnikov-Marjon E, Serda R, Pastuszyn A, Graham SE, Marcus CB, et al. Hybrid homology modeling and mutational analysis of cytochrome P450C24A1 (CYP24A1) of the Vitamin D pathway: insights into substrate specificity and membrane bound structure-function. Arch Biochem Biophys 2007;460:262-73.

116. Halbritter J, Baum M, Hynes AM, Rice SJ, Thwaites DT, Gucev ZS, et al. Fourteen monogenic genes account for $15 \%$ of nephrolithiasis/nephrocalcinosis. J Am Soc Nephrol 2015;26:543-51.

117. Lederer E, Wagner CA. Clinical aspects of the phosphate transporters NaPi-Ila and NaPi-Ilb: mutations and disease associations. Pflugers Arch 2019;471:137-48.

118. Oddsson A, Sulem P, Helgason H, Edvardsson VO, Thorleifsson $\mathrm{G}$, Sveinbjornsson $\mathrm{G}$, et al. Common and rare variants associated with kidney stones and biochemical traits. Nat Commun 2015;6:7975.

119. De Paolis E, Minucci A, De Bonis M, Scaglione GL, Gervasoni J, Primiano A, et al. A rapid screening of a recurrent CYP24A1 pathogenic variant opens the way to molecular testing for Idiopathic Infantile Hypercalcemia (IIH). Clin Chim Acta 2018;482:8-13.

120. Dusso AS, Gomez-Alonso C, Cannata-Andia JB. The hypercalcaemia of CYP24A1 inactivation: new ways to improve diagnosis and treatment. Clin Kidney J 2015;8:456-8.

121. Jones G, Kottler ML, Schlingmann KP. Genetic diseases of vitamin D metabolizing enzymes. Endocrinol Metab Clin North Am 2017;46:1095-117.

122. Hooman N. Bisphosphonates for treatment of severe idiopathic infantile hypercalcemia. Iran J Kidney Dis 2013;7:79-81.

123. Skalova S, Cerna L, Bayer M, Kutilek S, Konrad M, Schlingmann KP. Intravenous pamidronate in the treatment of severe idiopathic infantile hypercalcemia. Iran J Kidney Dis 2013;7:160-4.

124. Jacobs TP, Kaufman M, Jones G, Kumar R, Schlingmann KP, Shapses $\mathrm{S}$, et al. A lifetime of hypercalcemia and hypercalciuria, finally explained. J Clin Endocrinol Metab 2014;99:708-12.

125. Davidson Peiris E, Wusirika R. A case report of compound heterozygous CYP24A1 mutations leading to nephrolithiasis successfully treated with ketoconazole. Case Rep Nephrol Dial 2017;7:167-71.

126. Marks J, Debnam ES, Unwin RJ. Phosphate homeostasis and the renal-gastrointestinal axis. Am J Physiol Renal Physiol 2010;299:F285-96.

127. Sayers J, Hynes AM, Srivastava S, Dowen F, Quinton R, Datta $\mathrm{HK}$, et al. Successful treatment of hypercalcaemia associated with a CYP24A1 mutation with fluconazole. Clin Kidney J 2015;8:453-5.

128. Hawkes CP, Li D, Hakonarson H, Meyers KE, Thummel KE, Levine MA. CYP3A4 induction by rifampin: an alternative pathway for vitamin $D$ inactivation in patients with CYP24A1 mutations. J Clin Endocrinol Metab 2017;102:1440-6.

129. Huang J, Coman D, McTaggart SJ, Burke JR. Long-term follow-up of patients with idiopathic infantile hypercalcaemia. Pediatr Nephrol 2006;21:1676-80.

Supplementary Material: The online version of this article offers supplementary material (https://doi.org/10.1515/cclm-2018-1208). 